\title{
Análise fitoquímica e atividade fotoprotetora de extrato etanólico de Euphorbia tirucalli Linneau (Euphorbiaceae)
}

ORLANDA, J.F.F"; VALE, V.V. ${ }^{2}$

Universidade Estadual do Maranhão, Campus de Imperatriz (CESI-UEMA), Departamento de Química e Biologia, Laboratório de Biotecnologia Ambiental (LABITEC), 65901-480, Imperatriz - MA, Brasil. ${ }^{2}$ Faculdade de Imperatriz (FACIMP), 65912-901 Imperatriz - MA, Brasil. *Autor para correspondência: ffranca@cesi.uema.br

RESUMO: Euphorbia tirucalli Linneau, conhecida popularmente como "avelós", "cachorro pelado" ou "pau-pelado", é utilizada na medicina popular para o tratamento de câncer, antibiótico e antivirótico. As plantas medicinais são, frequentemente, utilizadas com o intuito de substituir ou auxiliar as terapias convencionais no tratamento de várias doenças, inclusive para evitar os malefícios causados por radiações ultravioletas. Com isso, o objetivo desta pesquisa foi avaliar o potencial fotoprotetor e a triagem fitoquímica do extrato etanólico das partes aéreas de avelós. A atividade fotoprotetora foi avaliada por meio de ensaios in vitro segundo o método adaptado de Mansur, utilizando extrato etanólico das partes aéreas. Os resultados obtidos mostraram que o estudo fitoquímico evidenciou a presença de açúcares redutores, alcaloides, fenóis, flavonoides, taninos, saponinas, esteroides e triterpenóides na fração etanólica. O extrato apresentou capacidade antioxidante $\left(E_{50}\right)$ de $16,98 \pm 0,35 \mu \mathrm{g} \cdot \mathrm{mL}^{-1}$, teor de compostos fenólicos de 96,18 $\pm 1,33 \mathrm{mg}$ EqAG/g e flavonoides de 63,57 $\pm 2,74 \mathrm{mg} \mathrm{EqC} / \mathrm{g}$ que podem favorecer a atividade fotoprotetora. Avaliando o fator de proteção solar, pode-se observar que todas as concentrações testadas $\left(0,01\right.$ a $\left.0,1 \mu \mathrm{g} \cdot \mathrm{mL}^{-1}\right)$ apresentaram potencial de fotoproteção da radiação ultravioleta com variação de FPS de 6,05 a 19,84, respectivamente. Esses resultados sugerem que o extrato etanólico de Euphorbia tirucalli Linneau apresenta possível efeito fotoprotetor.

Palavras-chave: Euphorbia tirucalli Linneau, avelós, atividade fotoprotetora

\begin{abstract}
Phytochemical analysis and photoprotective activity of theethanolic extract of Euphorbia tirucalli Linneau (Euphorbiaceae). Euphorbia tirucalli Linneau, popularly known as "avelos", "naked cock" or "naked dog", is used in popular medicinein order to treat cancer, antibiotic and antiviral diseases. Herbal medicines are often employed in order to assist or replace conventional therapies for the treatment of several diseases, including the prevention of the damage caused by ultraviolet radiation. Thus, the aim of this research was to evaluate the photoprotective potential and phytochemical screening of the ethanol extract of the aerial parts of avelos. The photoprotective activity was assessed by in vitrotrials according to the method adapted from Mansur, using the ethanol extract of the aerial parts. The results showed that the phytochemical study revealed the presence of reducing sugars, alkaloids, phenols, flavonoids, tannins, saponins, steroids and triterpenoids in ethanolic fraction. The extract showed antioxidant activity $\left(\mathrm{EC}_{50}\right)$ of 16,98 \pm $0,35 \mu \mathrm{g} \cdot \mathrm{mL}^{-1}$, phenolic contents of 96,18 $\pm 1,33 \mathrm{mg}$ EqAG/g and 63,57 $\pm 2,74$ flavonoids from $\mathrm{mg} \mathrm{EqC/g}$ that can advantage the photoprotective activity. Assessing the sun protection factor, it can be observed that all the tested concentrations $\left(0,01\right.$ to $\left.0,1 \mu \mathrm{g} \cdot \mathrm{mL}^{-1}\right)$ showed potential for photoprotection from ultraviolet radiation with a range of 6,05 to 19,84 FPS, respectively. These results suggest that the ethanol extract of Euphorbia tirucalli Linneau presents possible photoprotective effect.
\end{abstract}

Keywords: Euphorbia tirucalli Linneau, avelos, photoprotective activity 


\section{INTRODUÇÃO}

As plantas medicinais representam opção terapêutica de grande importância para a manutenção das condições de saúde das pessoas, especialmente, para a população de baixa renda, com o intuito de substituir ou auxiliar as terapias convencionais no tratamento de várias doenças, da facilidade de obtenção e do baixo custo (Lima et al., 2013).

As plantas medicinais apresentam ampla diversidade de metabólitos secundários com diferentes atividades biológicas, existindo a necessidade de um aprofundamento no conhecimento das propriedades farmacológicas das espécies vegetais e suas possíveis utilizações no desenvolvimento de novos medicamentos (Simões et al., 2010; Oliveira et al., 2008).

Entre as propriedades farmacológicas estudadas em produtos naturais está à ação fotoprotetora. A atividade fotoprotetora permite avaliar a habilidade dos princípios ativos extraídos de plantas em proteger a pele de eritemas, edemas, reduzir o risco de queimaduras e o risco de carcinoma de células da camada basal e espinhosa (Oliveira-Júnior et al., 2012; Santana et al., 2012; Polonini et al., 2011).

A fotoproteção começou a ser, extremamente, valorizada, não somente para a prevenção das queimaduras solares, mas em virtude dos seus efeitos preventivos contra os efeitos nocivos da radiação UVA e UVB deletérias às células, estruturas celulares e o desenvolvimento de cânceres de pele (Bissonette, 2008; Rigel, 2008; Rünger \& Kappes, 2008; Russo \& Halliday, 2006).

A espécie botânica Euphorbia tirucalli Linneau é uma planta pertencente à família Euphorbiaceae, representada por cerca de 300 gêneros e, aproximadamente 7500 espécies (Machado, 2007), conhecida popularmente como "avelós", "cachorro pelado", "pau-pelado", "cega-olho", entre outros nomes. Botanicamente, classificada como uma planta suculenta, produtora de um látex branco que mede até dez metros de altura, apresenta tronco e ramos lenhosos de cor marrom-claro ou acinzentado, do qual surgem os ramos jovens que são verdes e cilíndricos (ITF, 2008).

$\mathrm{Na}$ literatura, são descritos diversos usos populares como analgésico, anti-inflamatório, emético, laxativo, impotência, estimulante do sistema imune, tratamento de câncer, antiescorpiônica, ofídica, purgativo, antireumático, antiasmático, antiespasmódico, antibiótico, antivirótico, expectorante, fungicida, antissifilítico, tratamento de carcinomas -epiteliomas benignos e cauterizante de verrugas (Tofanelli \& Silva, 2011; Machado, 2007).

Considerando o desafio que a desco- berta de novos fármacos a partir de plantas, este trabalho teve como objetivos realizar uma triagem fitoquímica e avaliar a atividade fototoprotetora in vitro do extrato bruto etanólico de Euphorbia tirucalli Linneau.

\section{MATERIAL E MÉTODO Material botânico}

A espécie vegetal foi coletada no município de Imperatriz, estado do Maranhão, localizado na região Nordeste do Brasil, no período de junho a agosto de 2012. A identificação botânica da espécie vegetal em estudo foi identificada pelo botânico Marcelo Francisco da Silva, do Departamento de Química e Biologia da Universidade Estadual do Maranhão, Campus de Imperatriz (CESI-UEMA), como pertencente à família Euphorbiaceae, cujo nome científico é Euphorbia tirucalli Linneau e nome popular de "avelós", conforme características das folhas e galhos. A exsicata encontra-se depositada no herbário dessa instituição, sob registro $n^{\circ}$ 002/2012.

\section{Preparação do extrato etanólico}

Para obtenção do extrato das partes aéreas de Euphorbia tirucalli Linneau foi utilizado o processo de maceração. O material botânico, após limpeza, secagem em estufa de ar circulante a $40^{\circ} \mathrm{C}$ por 48 horas, foi triturado e pulverizado em moinho de facas elétrico (Tecnal, Modelo 680). Em seguida, $30 \mathrm{~g}$ do material vegetal foi submetido à maceração com álcool etílico na proporção de 5:1 (etanol/água) $(\mathrm{v} / \mathrm{v})$, permanecendo em contato por um período de quinze dias à temperatura ambiente e mantido ao abrigo da luz.

O extrato bruto etanólico (EBE) obtido, após filtração, foi concentrado à temperatura ambiente em evaporador rotativo e armazenado em frasco âmbar para posterior análise.

\section{Investigação fitoquímica qualitativa preliminar}

A triagem fitoquímica dos metabólitos secundários foi realizada no extrato etanólico bruto, segundo metodologias adaptadas de Barbosa et al. (2004) e Matos (1997). Os ensaios foram conduzidos para as seguintes classes químicas: cumarinas, terpenos, esteroides, alcaloides, taninos (inclusive taninos pirogálicos, taninos pirocatéquico, taninos gálicos), flavonoides e saponinas. A classificação dos grupos de metabólitos secundários foi realizada por meio de reações químicas que resultaram no desenvolvimento de cor e/ou formação de precipitado 


\section{Avaliação da atividade antioxidante Teor de fenólicos totais}

A determinação do teor de fenólicos totais presentes no EBE foi realizada empregando o reagente de Folin-Dennis, com o ácido gálico como composto padrão de acordo com o método de Slinkard \& Singleton (1977), modificado por Almeida et al. (2012). Neste ensaio foram utilizadas alíquotas de $0,5 \mathrm{~mL}$ dos extratos em diferentes concentrações, provenientes de uma solução estoque de $0,2 \mathrm{mg} \cdot \mathrm{mL}^{-1} \mathrm{em}$ etanol P.A., foram misturadas a $2,5 \mathrm{~mL}$ de reagente Folin-Dennis, com agitação por 1 minuto. Em seguida, 2,0 $\mathrm{mL}$ de $\mathrm{Na}_{2} \mathrm{CO}_{3}(14 \%)$ foram acrescentados à mistura e agitação por 1 minuto. Após 2 horas, a absorbância das amostras foi medida a $760 \mathrm{~nm}$. $O$ teor de fenólicos totais foi expresso como miligrama de equivalente de ácido gálico por grama de amostra (mg EAG/g), a partir da curva de calibração construída com o padrão de ácido gálico $(0,02$ a $\left.200 \mu \mathrm{g} \cdot \mathrm{mL}^{-1}\right)$ e todas as análises foram realizadas em triplicata.

\section{Teor de flavonoides totais}

O conteúdo de flavonoides totais foi determinado pelo método colorimétrico descrito por Dewanto et al. (2002) e Meda et al. (2005). Alíquotas de $2,0 \mathrm{~mL}$ de uma solução de $\mathrm{AlCl}_{3}$ a $2 \%$ foram adicionadas a $2,0 \mathrm{~mL}$ de soluções de extratos etanólicos $\left(0,4 \mathrm{mg} \cdot \mathrm{mL}^{-1} \mathrm{em}\right.$ etanol). Após 30 minutos de incubação, os valores de absorbância foram mensurados a $415 \mathrm{~nm}$, empregando-se etanol como branco. $O$ teor de flavonoides totais foi expresso como miligrama de equivalente de quercetina por grama de amostra (mg EQ/g), a partir da curva de calibração construída com o padrão quercetina $\left(0,02\right.$ a $\left.200 \mu \mathrm{g} \cdot \mathrm{mL}^{-1}\right)$. Todas as análises foram realizadas em triplicata.

\section{Atividade sequestradora do radical DPPH}

$A$ atividade antioxidante foi baseada no método de redução do radical 2,2'-difenil1-picrihidrazilo (DPPH.). Os resultados foram expressos como $\mathrm{EC}_{50}$, que é a quantidade de antioxidantes necessária para diminuir em $50 \%$ a concentração inicial de DPPH da solução. Dessa forma, quanto menor o valor do $\mathrm{EC}_{50}$, menor terá sido o valor do extrato utilizado para reduzir o radical DPPH• e maior a sua atividade antioxidante (Costa et al., 2010; Lima et al, 2010; Sousa et al., 2007; Mariutti \& Bragagnolo, 2007; Govidarajan et al., 2003).

A absorbância do radical DPPH foi determinada em $517 \mathrm{~nm}$ em espectrofotômetro Femton $800 \mathrm{XI}$. Em 1,5 mL de radical DPPH a $6 \times 10^{-5} \mu \mathrm{mol} . \mathrm{L}^{-1}$ foram dissolvidos em metanol puro, adicionada $0,5 \mathrm{~mL}$ das soluções contendo diferentes concentrações de cada extrato ou do controle positivo (quercetina) nas concentrações de 0,01 a $0,25 \mathrm{mg} \cdot \mathrm{mL}^{-1}$ e em seguida realizada a homogeneização e depois armazenada à temperatura ambiente. Após um período de 30 minutos, foram realizadas as leituras das absorbâncias das amostras e do branco $(\mathrm{MeOH}+$ DPPH) em intervalos de 5 minutos, determinando a curva cinética da reação. Todas as análises foram realizadas em triplicata.

A atividade sequestradora de radicais livres pelas amostras (AS\%) dos extratos nas diferentes concentrações foi calculado pela equação 1 :

AS $(\%)=\frac{(\text { A DPPH }- \text { A amostra })}{(\mathrm{ADPPH})} \times 100 \quad$ Equação 1

onde $A_{D P P H}$ é a medida da absorbância inicial da solução de DPPH, e A é a absorbância da mistura reacional (solução etanólica dos extratos nas diferentes concentrações e solução de DPPH) no tempo de 60 minutos.

Os ensaios foram realizados em triplicata e os resultados obtidos foram analisados no programa estatístico Origin 7.0. Neste programa as regressões lineares dos extratos foram obtidas de acordo com as suas diferentes concentrações em função dos percentuais de atividade sequestradora de radicais (AS\%). Na equação da reta: $y=a+b x, y$ representa $50 \%$ do sequestro do radical livre DPPH e $x$ é a concentração da amostra que sequestra $50 \%$, ou seja a concentração eficiente $\left(\mathrm{EC}_{50}\right)$ das amostras analisadas. Os valores de $\mathrm{EC}_{50}$ foram expressos como média \pm erro padrão.

\section{Avaliação da atividade fotoprotetora}

A atividade fotoprotetora foi avaliada in vitro através da determinação do comprimento de onda máximo $\left(\lambda_{\text {maxximo. }}\right)$ e da absorbância máxima $\left(A_{\text {máximaa }}\right)$. Os extratos secos foram diluídos em álcool etílico PA (Merck) $\left(0,01\right.$ a $\left.0,1 \mu \mathrm{g} \cdot \mathrm{mL}^{-1} \mathrm{p} / \mathrm{v}\right)$ e realizada varredura entre os comprimentos de onda de 260 a $400 \mathrm{~nm}$ (Espectrofotômetro FEMTO, modelo 800XI, em cubeta de quartzo de $1,0 \mathrm{~cm}$ caminho óptico), para verificar a absorção nas regiões ultravioleta $A$ e $B$ (UVA e UVB). O álcool etílico absoluto PA foi utilizado como branco e o experimento realizado em triplicata.

\section{Cálculo do Fator de Proteção Solar (FPS)}

Os cálculos foram realizados considerando os intervalos de comprimento de onda $(\lambda)$ determinados por Mansur et al. (1986a; 1986b). Os valores do efeito eritemogênico da radiação - $E E(\lambda)$ e intensidade do sol - I $(\lambda)$ utilizados para o cálculo do FPS foram os mesmos usados na literatura, empregando a equação 2 : 
FPS Espectrofotométrico=FC. $\sum_{290}^{320} \mathrm{EE}(\lambda) .1(\lambda) \cdot \operatorname{Abs}(\lambda) \quad$ Equação 2

Onde: $F C=$ fator de correção (10), referente $a$ um FPS $=4$; $\operatorname{EE~}(\lambda)=$ efeito eritemogênico da radiação; I $(\lambda)=$ intensidade do sol; abs $(\lambda)=$ leitura espectrofotométrica da absorbância do extrato etanólico de avelós.

\section{RESULTADO E DISCUSSÃO}

A triagem fitoquímica da espécie Euphorbia tirucalli Linneau, realizada na fração etanólica das partes aéreas da espécie vegetal em estudo, através de reações químicas cromogênicas ou de precipitação, indicou presença de açúcares redutores, alcaloides, compostos fenólicos, flavonoides, taninos, saponinas, esteroides e triterpenóides, como mostra a Tabela 1.

TABELA 1. Constituintes químicos do extrato etanólico das partes aéreas de Euphorbia tirucalli Linneau.

\begin{tabular}{lc}
\hline Classes de constituintes químicos & Resultados $^{\mathrm{a}}$ \\
\hline Alcaloides & + \\
Açúcares redutores & + \\
Antraquinonas & - \\
Compostos fenólicos & + \\
Cumarinas & - \\
Esteroides & + \\
Flavonoides & + \\
Saponinas & + \\
Taninos & + \\
Triterpenos & + \\
\hline
\end{tabular}

apresença (+) ou ausência (-)

Com o objetivo de estimar a presença de compostos com capacidade redutora, foi realizado o ensaio de quantificação do conteúdo de compostos fenólicos totais e flavonoides no EBE. De acordo com os resultados obtidos no ensaio do sequestro do DPPH, o extrato apresentou capacidade antioxidante $\left(\mathrm{EC}_{50}\right)$ de 16,98 $\pm 0,35$ $\mu \mathrm{g} \cdot \mathrm{mL}^{-1}$, enquanto o controle positivo quercetina foi de $0,64 \pm 0,70 \mu \mathrm{g} \cdot \mathrm{mL}^{-1}$. Os resultados encontramse abaixo dos descrito por Campos et al. (2003), que considera os extratos botânicos ativos quando apresentam $\mathrm{EC}_{50}<500 \mu \mathrm{g} \cdot \mathrm{mL}^{-1}$.

A atividade sequestradora de radicais livres para o EBE pode ser explicada pela presença de compostos fenólicos $(96,18 \pm 1,33 \mathrm{mg} \mathrm{EqAG/g})$ e de flavonoides $(63,57 \pm 2,74 \mathrm{mg} \mathrm{EqC} / \mathrm{g})$, um valor considerado alto quando comparado com outras espécies botânicas descritas na literatura.
Dessa forma, é possível afirmar que os compostos fenólicos, especialmente flavonoides, podem ser os responsáveis pela ação antioxidante do $E B E$, tendo em vista os inúmeros trabalhos que relatam as diferentes atividades exibidas por esta classe de compostos (Silva et al., 2010; Sousa et al., 2007; Violante et al., 2008; Alves et al., 2010; Souza et al., 2005; Cunha, 2005).

A excelente correlação observada entre o teor de fenólicos totais e a atividade antioxidante do EBE, avaliada pela atividade sequestradora do radical livre DPPH $\left(R^{2}=0,99\right.$, indica que as substâncias fenólicas presentes no extrato são responsáveis pela boa atividade antioxidante desta espécie.

O potencial antioxidante, teor de fenólicos totais e flavonoides também podem ser úteis para promover outras investigações e correlacionar esta atividade a outras importantes, como, por exemplo, à atividade fotoprotetora.

A atividade fotoprotetora do EBE fol avaliada mediante as análises espectrofotométricas em diferentes concentrações na faixa do ultravioleta (200 a 400 nm), como mostra a Figura 1.

Os resultados apresentados na Figura 1 são um indicativo para a utilização do extrato etanólico de "avelós" em produtos fotoprotetores, uma vez que a faixa de radiação ultravioleta situase nos comprimentos de ondas entre 290 a 400 $\mathrm{nm}$ e a absorbância encontrada nessa faixa foram, relativamente, altas.

A presença de flavonoides, taninos e alcaloides no EBE assume uma posição de destaque em termos da absorção espectral pela radiação solar na faixa do UV-B com finalidade de antienvelhecimento e de fotoproteção (Ramos et al., 2010; Dal'Belo, 2008; Gobbo-Neto \& Lopes, 2007). Além de ser, um indicativo de absorção da radiação UV, proporcionando altas absortividades molares $\left(\varepsilon / \mathrm{M}^{-1} \mathrm{~cm}^{-1}\right)$ para comprimentos de onda $(\lambda)$ que culminem com um FPS biologicamente efetivo (Simões et al., 2010).

A faixa espectral apresentada na Figura 1, com máximo em $305 \mathrm{~nm}$, é a de maior relevância em termos de fotoatividade, pois é a faixa onde ocorre a maior indução a quebra de dímeros de ciclobutanopiridina encontrado no DNA epidermal, conforme descrito no relatório da Organização Mundial da Saúde (WHO) e Rosa et al. (2008).

A avaliação do fator de proteção solar (FPS) foi determinada pelo cálculo proposto por Mansur (1986a; 1986b) que relaciona a absorbância do extrato com o efeito eritematógeno da radiação e a intensidade da luz em comprimentos de ondas determinados entre 290 a $320 \mathrm{~nm}$ (espectro específico da região UVB). O FPS, estimado por

Rev. Bras. PI. Med., Campinas, v.17, n.4, supl. I, p.730-736, 2015. 


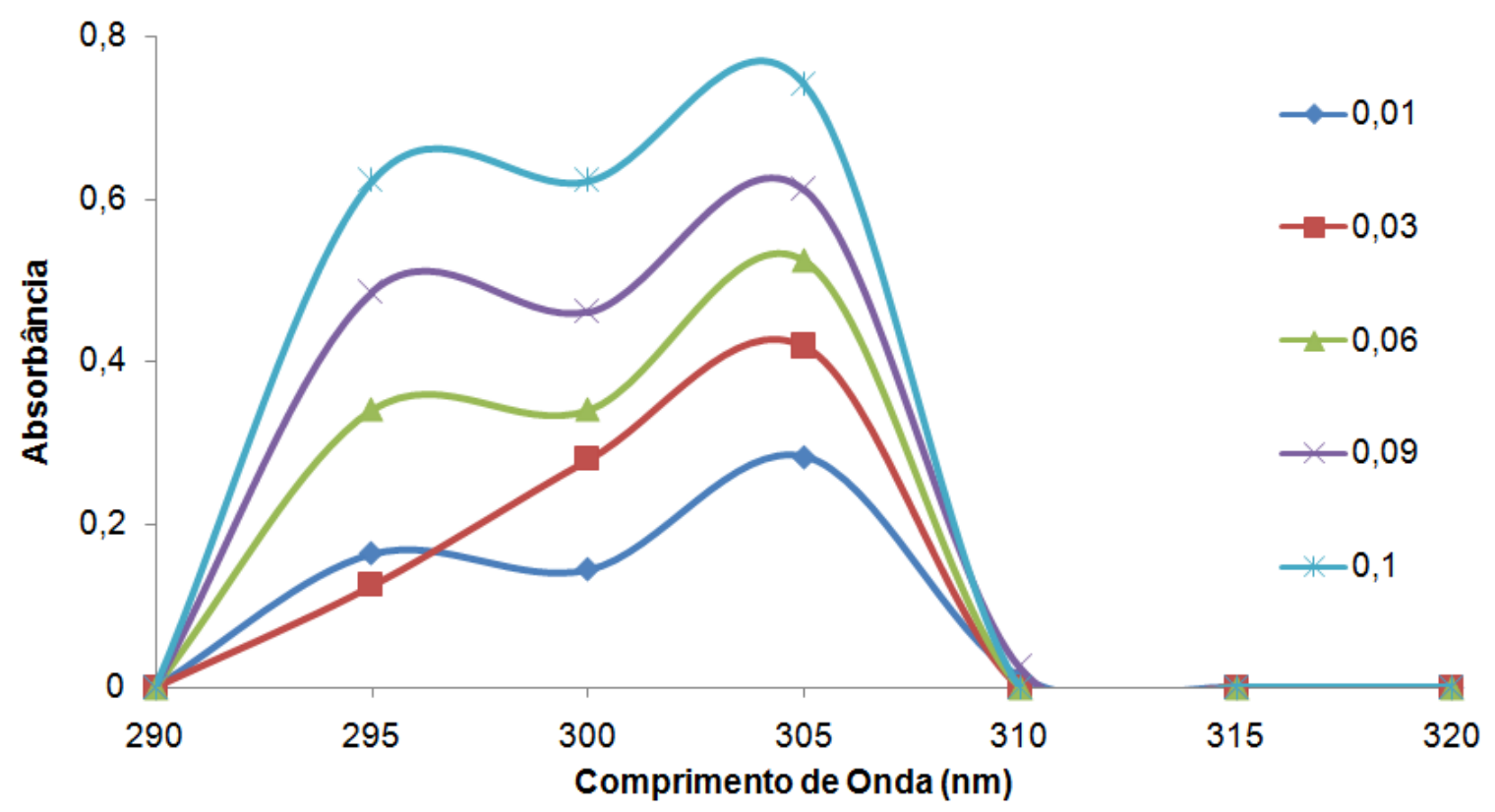

FIGURA 1. Análise espectrofotométrica do extrato etanólico de "avelós" em diferentes concentrações.

espectrofotometria, avalia a substância em estudo, de acordo com a altura, largura e localização da sua curva de absorção dentro do espectro do ultravioleta.

Apesar de ser um teste in vitro, já foi demonstrado que esta metodologia apresenta uma boa correlação com os testes in vivo (Ferrari, 2002; Santos et al., 1999), o que garante a confiabilidade das determinações analíticas.

Os resultados para o cálculo do FPS do extrato etanólico de "avelós", em diferentes concentrações, são apresentados na Tabela 2.

Avaliando o fator de proteção solar apresentado na Tabela 2, pode-se observar que todas as concentrações testadas apresentaram potencial de fotoproteção da radiação ultravioleta. $\mathrm{Na}$ concentração de $0,1 \mu \mathrm{g} \cdot \mathrm{mL}^{-1}$ do extrato apresentou FPS de 19,84, ou seja, protege a pele, praticamente, 20 vezes mais do que não utilizando o extrato.

Estes resultados podem ser explicados através das observações de diferentes pesquisadores que demonstraram a eficácia da atividade fotoprotetora, depende da capacidade de absorção de energia radiante atribuída aos grupos cromóforos presentes nos extratos de plantas, que é proporcional à sua concentração, intervalo de absorção e comprimento de onda onde ocorre absorção máxima (Violante et al., 2008; Maillan et al., 2005; Ribeiro et al., 2004; SIlva Filho et al., 2003).

Estes resultados encontram-se de acordo com a literatura, pois outros trabalhos demonstraram que diferentes produtos vegetais apresentaram absorção da UV e potencial para serem considerados filtros solares vegetais (Polonini et al., 2011; Nascimento et al., 2009; Di Mambro \& Fonseca, 2006; Souza et al., 2005; Goossens \& Lepoittevin, 2003).

Levando em conta o fator de proteção solar

TABELA 2. FPS do extrato etanólico de "avelós" em diferentes concentrações.

\begin{tabular}{lllllll}
\hline & & \multicolumn{2}{l}{ Concentrações $\left(\mu \mathrm{g} \cdot \mathrm{mL}^{-1}\right)$} & & \\
\cline { 2 - 7 }$N / \mathbf{n m}$ & EE x I & 0,01 & 0,03 & 0,06 & 0,09 & 0,1 \\
\hline 290 & 0,0150 & 0 & 0 & 0 & 0 & 0 \\
295 & 0,0817 & 0,164 & 0,125 & 0,341 & 0,485 & 0,622 \\
300 & 0,2874 & 0,145 & 0,28 & 0,341 & 0,461 & 0,622 \\
305 & 0,3278 & 0,283 & 0,419 & 0,524 & 0,611 & 0,74 \\
310 & 0,1864 & 0,013 & 0 & 0 & 0,025 & 0 \\
315 & 0,0839 & 0 & 0 & 0 & 0 & 0 \\
320 & 0,0180 & 0 & 0 & 0 & 0 & 0 \\
TOTAL & 1,0002 & 0,605 & 0,824 & 1,206 & 1,582 & 1,982 \\
FPS & & 6,05 & 8,24 & 12,06 & 15,82 & 19,82 \\
\hline
\end{tabular}

Rev. Bras. PI. Med., Campinas, v.17, n.4, supl. I, p.730-736, 2015. 
(FPS), determinado pela RDC 30, de 01 de junho de 2012, da Agência Nacional de Vigilância Sanitária (ANVISA, 2012), todas as concentrações do extrato etanólico testadas $\left(0,01\right.$ a $\left.0,1 \mu \mathrm{g} \cdot \mathrm{mL}^{-1} \mathrm{p} / \mathrm{v}\right)$ poderiam ser utilizadas, como protetores solares, pois o fator mínimo de proteção segundo essa resolução, é de FPS 06.

Os resultados obtidos nessa pesquisa são melhores que outros encontrados na literatura. Em estudos realizados por Rosa et al. (2008) utilizando o mesmo método, foram encontrados FPS 8 para mil-folhas (Achillea millefolium), 6 para repolho (Brassica oleracea var. capitata), 5 para tiririca (Cyperus rotundus), 5 para boldobrasileiro (Plectranthus barbatus), 2 para arnica paulista (Porophyllum ruderale (jacq.) Cass) e 1 para serralha (Sonchus oleraceus).

Os resultados da avaliação da atividade do extrato etanólico das partes aéreas de Euphorbia tirucalli Linneau demonstraram efeito fotoprotetor, no modelo experimental utilizado por Mansur nas concentrações testadas, sugerindo possível aplicação para fins terapêuticos em preparações dermatológicas.

\section{REFERÊNCIAS}

ALMEIDA, J.R.G.S. et al. Antinociceptive activity of ethanol extract from Duguetia chrysocarpa Maas (Annonaceae). The Scientific World Journal, v.2012, p.1-6, 2012.

ALVES, C.Q. et al. Métodos para determinação de atividade antioxidante in vitro em substratos orgânicos. Química Nova, v.33, n.10, p.2.202-2.210, 2010.

ANVISA, Agência Nacional de Vigilância Sanitária. Regulamento Técnico Mercosul sobre Protetores Solares em Cosméticos - RDC 30 de $1^{\circ}$ de Junho de 2012.

BARBOSA, W.L.R. et al. Manual para análise fitoquímica e cromatográfica de extratos vegetais. 2 ed. Revista Científica da UFPA, v.4, 2004. Disponível em: http:// http://www2.ufpa.br/rcientifica/didaticos_cientificos/ pdf_textos/abord_fitoquimica.pdf. Acesso em: 15 agosto 2014.

BISSONETTE, R. Update on Sunscreens. Skin Therapy Letter v.13, n.1, p.5-7, 2008.

CAMPOS, M.G. et al. Age-induced diminution of free radical scavenging capacity in bee pollens and the contribution of constituent flavonoids. Journal of Agricultural and Food Chemistry, v.51, p.742-745, 2003.

COSTA, D.A. et al. Constituintes químicos, fenóis totais e atividade antioxidante de Sterculia striata St. Hil. et Naudin. Acta Amazica, v.40, n.1, p.207-212, 2010.

CUNHA, A.P. Farmacognosia e Fitoquímica. Lisboa: Fundação Calouste Gulbenkian, 2005.

DAL'BELO, S.E. Avaliação da eficácia fotoprotetora, penetração cutânea e segurança de formulações cosméticas contendo extratos de chá verde e Ginkgo biloba. 2008. 192f. Tese (Doutorado em Ciências Farmacêuticas) - Universidade de São Paulo,
São Paulo

DEWANTO, V. et al. Processed sweet corn has higher antioxidant activity. Journal of Agricultural and Food Chemistry, v.50, n.10, p.3.010-3.014, 2002.

DI MAMBRO, V.M.; FONSECA, M.J.V. Avaliação da eficácia fotoprotetora in vivo de formulações contendo extratos de Ginkgo biloba e Glycirrhiza glabra. In: XX CONGRESSO BRASILEIRO DE COSMETOLOGIA. São Paulo, Brasil. 2006.

WORLD HEALTH ORGANIZATION (WHO). ECH 160: Ultraviolet radiation, Geneva: WHO; 1994, 353p.

FERRARI, M. Desenvolvimento e avaliação da eficácia fotoprotetora de emulsões múltiplas contendo metoxicinamato de etilexila e óleo de andiroba (Carapa guyanensis). 2002. 142f. Tese (Doutorado em Ciências Farmacêuticas) Universidade de São Paulo, Faculdade de Ciências Farmacêuticas de Ribeirão Preto.

GOBBO-NETO, L.; LOPES, N.P. Plantas medicinais: fatores de influência no conteúdo de metabólitos secundários. Química Nova, v.30, n.2, p.374-381, 2007.

GOOSSENS, A.; LEPOITTEVIN; J.P. Allergie de contact aux cosmétiques et aux composants de perfums: aspects cliniques, chimiques et diagnostiques nouveaux. Revue Française D'allergologie et D'immunologie Clinique, v.43, p.294-300, 2003.

GOVIDARAJAN, R. et al. Studies on the antioxidant activities of Desmodium gangeticum. Biological \& Pharmaceutical Bulletin, v.26, p.1424-7, 2003.

LIMA, A. ÍNDICE TERAPÊUTICO FITOTERÁPICO: ITF, 1ed. - Petrópolis, RJ: EPUB, 2008. 328p.

LIMA, L.A.R.S. et al. Acetogenins from Annona cornifolia and their antioxidant capacity. Food Chemistry, v.122, n.4, p.1.129- 1.138, 2010.

LIMA, L.R. et al. Avaliação da atividade antiedematogênica, antimicrobiana e mutagênica das sementes de Amburana cearensis (A. C. Smith) (Imburana-decheiro). Revista Brasileira de Plantas Medicinais, Campinas, v.15, n.3, p.415-422, 2013.

MACHADO, M. M. Perfil fitoquímico e avaliação dos principais efeitos biológicos e imunológicos in vitro da Euphorbia tirucalli L. 2007. 105 p. Tese (Mestrado do Programa de Pós-graduação em Ciências Farmacêuticas). Universidade Federal de Santa Maria: Santa Maria - RS.

MAILLAN, P. et al. Protecting against UV - induced degradation and enchancing shine. Cosmet Toiletries, v.120, p. 65-71, 2005.

MANSUR, J.S. et al. Correlação entre a determinação do fator de proteção solar em seres humanos e por espectrofotometria. Anais Brasileiros de Dermatologia, v.61, p.67-172, 1986a.

MANSUR, J.S. et al. Determinação do fator de proteção solar por espectrofotometria. Anais Brasileiros de Dermatologia, v.61, p. 121-124. 1986b.

MARIUTTI, L.R.B.; BRAGAGNOLO, N. Revisão: Antioxidantes Naturais da Família Lamiaceae Aplicação em Produtos Alimentícios. Brazilian Journal of Food Technology, v.10, n.2, p.96-103, 2007.

MATOS, F. J. A. Introdução a fitoquímica experimental. 2.ed. Fortaleza: UFC, 1997. 141p.

MEDA, A. et al. Determination of the total phenolic, flavonoid and proline contents in Burkina Fasan honey,

Rev. Bras. PI. Med., Campinas, v.17, n.4, supl. I, p.730-736, 2015. 
as well as their radical scavenging activity. Food Chemistry, v.91, p.571-577, 2005.

NASCIMENTO, C.S. et al. Incremento do FPS em formulação de protetor solar utilizando extratos de própolis verde e vermelha. Revista Brasileira de Farmácia, v.30, n.1, p.334-339, 2009.

OLIVEIRA, R.B. et al. Avaliação dos efeitos depressores centrais do extrato etanólico das folhas de Synadenium umbellatum Pax. e de suas frações em camundongos albinos. Revista Brasileira de Ciências Farmacêuticas Brazilian Journal of Pharmaceutical Sciences, v.44, n.3, p.485-491, 2008.

OLIVEIRA-JÚNIOR, R.G. et al. Phytochemical screening, antioxidant and antibacterial activity of extracts from the flowers of Neoglaziovia variegata (Bromeliaceae). Journal of Chemical and Pharmaceutical Research, v.4, n.10, p. 4489-4494, 2012.

POLONINI, H.C. et al. Fotoprotetores naturais como instrumento de ação primária na prevenção do câncer de pele. Revista de Atenção Primária a Saúde, v.14, n.2, p.216-223, 2011.

RAMOS, M.F.S. et al. Avaliação da atividade antissolar e estudos preliminares de fotodegradação da própolis. Revista Fitos, v.5, n.3, 2010.

RIBEIRO, R.P. et al. Avaliação do fator de proteção solar (FPS) in vitro de produtos comerciais e em fase de desenvolvimento. Infarma, v.16, n. 7-8, 2004.

RIGEL, D.S. Cutaneous ultraviolet exposure and its relationship to the development of skin cancer. Journal of American Academy of Dermatology, v.58, n.1, p.129-132, 2008.

ROSA, M.B. et al. Estudo Espectrofotométrico da atividade fotoprotetora de extratos aquosos de Achillea millefolium, Brassica oleracea Var. capitata, Cyperus rotundus, Plectranthus barbatus, Porophyllum ruderale (Jacq.) Cass e Sonchus oleraceus. Revista Eletrônica de Farmácia, v.5, n.1, p.101-110, 2008.

RÜNGER, T.M; KAPPES, U.P. Mechanisms of mutation formation with long-wave ultraviolet light (UVA). Photodermatology, Photoimmunology and
Photomedicine, v.24, n.1, p.2-10, 2008.

RUSSO, P.A.J.; HALLIDAY, G.M. Inhibition of nitric oxide and reactive oxygen species production improves the ability of a sunscreen to protect from sunburn, immunosuppression and photocarcinogenesis. British Journal of Dermatology, v.155, n.1, p.408-415, 2006.

SANTANA, C.R.R. et al. Phytochemical screening, antioxidant and antibacterial activity of Encholirium spectabile (Bromeliaceae). International Journal of Sciences, v.1, n.11, p.1-19, 2012.

SANTOS, E.P. et al. In vitro and in vivo determinations of sun protection factors of sunscreen lotions with octylmethoxycinnamate. International Journal of Cosmetic Science, v.21, p.1-5, 1999.

SILVA FILHO, E.A. et al. Moléculas inibidoras de radiações UV. Cosmet Toiletries, v.15, p.82-84, 2003.

SILVA, M.L.C. et al. Compostos fenólicos, carotenoides e atividade antioxidante em produtos vegetais. Semina: Ciências Agrárias, v.31, n.3, p.669-682, 2010.

SIMÕES, C.M.O. et al. Farmacognosia: da planta ao medicamento. 6. ed. Porto Alegre: Editora da UFRGS, 2010. 1102p.

SLINKARD, K.; SINGLETON, V.L. Total phenol analyses automation and comparison with manual methods. American Journal of Enology and Viticulture, v.28, p.49-55, 1977.

SOUSA, C.M.M. et al. Fenóis totais e atividade antioxidante de cinco plantas medicinais. Química Nova, v.30, n.2, p.351-355, 2007.

SOUZA, T.M. et al. Avaliação da atividade fotoprotetora de Achillea millefolium L. Revista Brasileira de Farmacognosia, v.15, n.1, p.36-38, 2005.

TOFANELLI, E.J.; SILVA, F.A. Propriedades fitoterápicas de Euphorbia tirucalli L.: da etnobotânica a farmacognosia. BIOFAR, v.6, n.1, p.151-166, 2011.

VIOLANTE, I.M.P. et al. Estudo preliminar da atividade fotoprotetora in vitro de extratos vegetais do cerrado de Mato Grosso. Revista Brasileira de Farmacognosia, v.89, n.3, p.175-179, 2008.

Rev. Bras. PI. Med., Campinas, v.17, n.4, supl. I, p.730-736, 2015. 